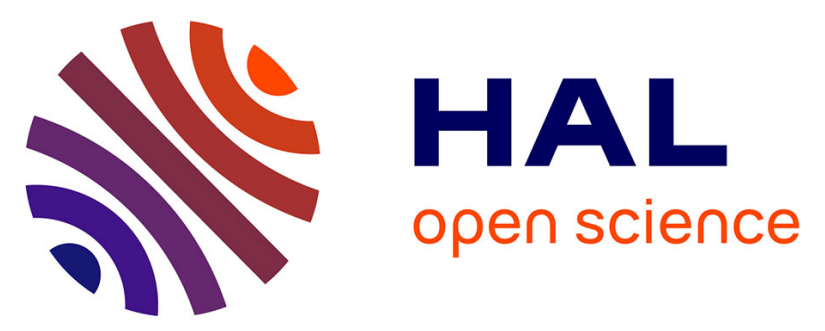

\title{
RECAST: Telling Apart Social and Random Relationships in Dynamic Networks
}

Pedro O.S. Vaz de Melo, Aline Carneiro Viana, Marco Fiore, Katia Jaffrès-Runser, Frédéric Le Mouël, Antonio A. F. Loureiro

\section{- To cite this version:}

Pedro O.S. Vaz de Melo, Aline Carneiro Viana, Marco Fiore, Katia Jaffrès-Runser, Frédéric Le Mouël, et al.. RECAST: Telling Apart Social and Random Relationships in Dynamic Networks. 16th ACM International Conference on Modeling, Analysis and Simulation of Wireless and Mobile Systems (MSWiM '13), Nov 2013, Barcelona, Spain. pp.327-334, 10.1145/2507924.2507950 . hal-00881804

\section{HAL Id: hal-00881804 https://hal.inria.fr/hal-00881804}

Submitted on 9 Nov 2013

HAL is a multi-disciplinary open access archive for the deposit and dissemination of scientific research documents, whether they are published or not. The documents may come from teaching and research institutions in France or abroad, or from public or private research centers.
L'archive ouverte pluridisciplinaire HAL, est destinée au dépôt et à la diffusion de documents scientifiques de niveau recherche, publiés ou non, émanant des établissements d'enseignement et de recherche français ou étrangers, des laboratoires publics ou privés. 


\section{RECAST: Telling Apart Social and Random Relationships in Dynamic Networks}

\author{
Pedro Olmo Vaz de Melo \\ Universidade Federal de \\ Minas Gerais, Brazil \\ olmo@dcc.ufmg.br \\ Katia Jaffrès-Runser \\ University of Toulouse, \\ IRIT, France \\ kjr@enseeiht.fr
}

\author{
Aline C. Viana \\ INRIA, France \\ aline.viana@inria.fr \\ Frederic Le Mouël \\ INSA Lyon, France \\ frederic.le-mouel@insa- \\ lyon.fr
}

\author{
Marco Fiore \\ IEIIT - CNR, Italy \\ marco.fiore@ieiit.cnr.it \\ Antonio A. F. Loureiro
Universidade Federal de
Minas Gerais, Brazil
loureiro@dcc.ufmg.br
}

\begin{abstract}
In this paper, we argue that the ability to accurately spot random and social relationships in dynamic networks is essential to network applications that rely on human routines, such as, e.g., opportunistic routing. We thus propose a strategy to analyze users' interactions in mobile networks where users act according to their interests and activity dynamics. Our strategy, named Random $r E$ lationship ClASsifier sTrategy (RECAST), allows classifying users' wireless interactions, separating random interactions from different kinds of social ties. To that end, RECAST observes how the real system differs from an equivalent one where entities' decisions are completely random. We evaluate the effectiveness of the RECAST classification on real-world user contact datasets collected in diverse networking contexts. Our analysis unveils significant differences among the dynamics of users' wireless interactions in the datasets, which we leverage to unveil the impact of social ties on opportunistic routing.
\end{abstract}

\section{Categories and Subject Descriptors}

H.2.8 [Database Management]: Database Applications-Data Mining; I.6.4 [Simulation and Modeling]: Model Validation and Analysis

\section{Keywords}

dynamic social networks; edge classification

\section{INTRODUCTION}

Recent studies have analyzed data generated from mobile individuals in urban regions, such as cab drivers in San Francisco (USA) [1] or students in large campuses [2, 3]. Particular attention has been paid to the dynamics of user movement, whose real-world complexity cannot be fully captured through synthetic models. Indeed, understanding user mobility is of fundamental importance

Permission to make digital or hard copies of all or part of this work for personal or classroom use is granted without fee provided that copies are not made or distributed for profit or commercial advantage and that copies bear this notice and the full citation on the first page. Copyrights for components of this work owned by others than ACM must be honored. Abstracting with credit is permitted. To copy otherwise, or republish, to post on servers or to redistribute to lists, requires prior specific permission and/or a fee. Request permissions from permissions@ acm.org. MSWiM'13, November 3-8, 2013, Barcelona, Spain.

Copyright 2013 ACM 978-1-4503-2353-6/13/11 ...\$15.00.

http://dx.doi.org/10.1145/2507924.2507950. when designing new communication protocols that exploit opportunistic encounters among users. In this case, the problem mainly lies in correctly forecasting future contacts. To that end, the regularity of daily activities comes in handy, as it enforces periodic (and thus predictable) space-time patterns in human mobility [4]. Building on such a feature, protocols have been designed to estimate the potential of mobile users to act as data forwarders, mainly leveraging complex network analysis metrics such as centrality measures.

Although human behavior is characterized by an elevated rate of regularity, random events are always possible in the routines of individuals. Those are hardly predictable situations that deviate from the regular pattern and are unlikely to repeat in the future; they originate from the fact that users are reasonable beings, whose connections represent decisions they take based on their personal motivations [5], which may also change over time. We refer to contact networks deriving from systems where users are reasonable beings as Dynamic Complex Wireless Networks (DCWN). For instance, a contact network composed of wireless hand-held devices is a clear example of DCWN, since the user mobility creates neither purely regular nor purely random connections among the entities composing the network.

The random events in DCWNs veil the ordinary patterns by introducing a significant amount of noise, thus making the process of knowledge discovery in such datasets a complex task. Therefore, the ability to accurately identify random and social events in DCWNs is essential to social analysis and to applications that rely on a precise description of human routines, such as recommendation systems, forwarding strategies and opportunistic dissemination protocols.

In this paper, we propose a Random rElationship ClASsifier sTrategy (RECAST) to classify relationships among users so as to spot contacts due to random events in DCWNs. To that end, RECAST examines how the real system would evolve if users' decisions were random. More precisely, we use the temporal graph originated from the real network dataset and a random counterpart of the same to tell apart edges representing random events from those created by actual social relationships, such as friendship or professional interactions. RECAST has a single and intuitive parameter, which makes it preferable to conventional methods for filtering and cleaning social network data that require arbitrary thresholds, many parameters, and often a deep knowledge of the system.

In summary, the contribution of this paper is threefold: 
- We introduce RECAST, a simple yet very effective way of classifying wireless contacts by leveraging metrics that reflect two major features of social networks: frequent user encounters and shared acquaintances.

- We unveil the large differences among contact datasets, and claim that conclusions drawn from evaluating a single dataset should not be generalized; rather, the validation of networking protocols or services has to consider different types of datasets.

- We show that the knowledge of the wireless relationship a pair of users share can be leveraged for the design of opportunistic routing schemes.

The paper is organized as follows. Section 2 discusses the related work. Section 3 details the design behind RECAST. Section 4 shows how we model real-world network traces into complex temporal networks. Section 5 details the RECAST implementation. Section 6 shows how RECAST can improve opportunistic routing solutions. Finally, conclusions are drawn in Section 7.

\section{RELATED WORK}

Social network analysis builds on the high predictability of human behaviors [6], which are mostly driven by regular, routine activities. As a consequence, connections among social network nodes can be modeled by mechanisms such as preferential attachment [7] and triangle formation [5], that leverages the existence of communities or highly connected hubs [8] in the network. This makes social networks different from random ones, such as the Erdős and Rényi network [9], where node connections are purely stochastic, being determined by a constant probability.

Given this predictability, social ties have been widely exploited in opportunistic mobile networks so as to favor network services. The considered problems range from multi-hop message forwarding [10] and multicasting [11], to network security [12]. To the best of our knowledge, the works of Miklas et al. [13] and Zyba et al. [14] are those most closely related to ours. These studies differ in that they classify either users or their interactions (i.e., vertices and edges in the social graph), respectively. Zyba et al. distinguish social and vagabond users according to their social mobility behavior. They analyze regularity of appearance and duration of visits in a given area of traces to sort out users. Hence, the resulting classification only works on a per-individual per-area basis. Miklas et al. classify links between friends and strangers. They assume that frequent pairwise node encounters represent friendship interactions, and empirically decide that pairs of users meeting 10 days or more out of 101 days are friends, whereas others are strangers.

Overall, our work extends the investigations in $[13,14]$ in the following ways. First, we propose a finer grained classifier, able not only to clearly characterize random interactions, but also to identify different kinds of social interactions: Friends, Acquaintances and Bridges. As such, we go a step further than [13] as we are able to identify edges corresponding to, e.g., familiar strangers, as defined by Milgram [15]: indeed, the users sharing a bridge interaction repeatedly encounter but may never experience an explicit social relationship. In addition, unlike the proposal by Zyba et al. [14], our strategy has no geographical dependency, i.e., it is of general validity.

\section{RATIONALE}

We consider social networks composed of individuals who are wirelessly connected over time. Such wireless encounters in these networks are driven by behaviors that tend: (i) to be regular and to repeat periodically; (ii) to build persistent communities of individuals or to generate commons acquaintances between them. The classification strategy we present in this paper leverages these two behaviors to efficiently distinguish social from random encounters in DCWNs. In the following, we detail our methodology and present the real world datasets considered in our analysis.

Social vs. random interactions: In DCWNs, interactions among the system entities are usually a consequence of semi-rational decisions. We say "usually" and "semi-rational" decisions because any system is subject to random events and irrational choices. Nevertheless, because most of the interactions still arise from conscious decisions made by their entities, the evolution of DCWNs is significantly different from the evolution of random networks, e.g., Erdős and Rényi networks [9]. Indeed, while in DCWNs the edges are created from semi-rational decisions, which tend to be regular and to repeat over time, in a random network the edges are created independently of the attributes of the network entities, i.e., the probability of connecting any two entities is always the same.

More formally, an individual may execute a social decision, or a random decision. Intuitively, if its probability of performing a social decision is greater than its probability of a random one, the network evolves to a well-structured social network. If the opposite it true, the network evolves as a random network, such as the Erdös and Rényi one.

Differentiating social from random network: The second major feature of DCWNs that we exploit in our study is the presence of communities, i.e., groups of individuals who are strongly connected to each other because they share the same interests or activity dynamics [5]. In contrast, communities can not be found in random networks where, as previously stated, edges are created stochastically and independently of the attributes of each node.

The network clustering coefficient has been widely used to discriminate random from social networks. Given an undirected graph $G(V, E)$ (where $V$ represents the set of network graph nodes, e.g., individuals, and $E$ is the set of links describing relationships among entities, e.g., contacts among individuals), the clustering coefficient $c c_{i}$ measures the probability that two neighbors of a node $i$ are also connected among them. Formally, it is calculated as $c c_{i}=2\left|E_{i}\right| /\left|N_{i}\right|\left(\left|N_{i}\right|-1\right)$, where $N_{i}$ is the set of neighbors of $i$, $E_{i}$ is the set of edges between nodes in $N_{i}$ and $|\cdot|$ is the cardinality of the included set. The clustering coefficient $\overline{c c}$ of the whole network is the average of all node clustering coefficients $c c_{i}, \forall i \in V$.

By introducing the equivalent random network $G^{R}$ as the random network constructed with the same number of nodes, edges and empirical degree distribution of its real world counterpart $G$, Watts and Strogatz [16] show that the clustering coefficient of a social network $G$ is one order of magnitude higher than the clustering coefficient of $G^{R}$. Thus, when a given network $G$ exhibits a clustering coefficient that is significantly (i.e., orders of magnitude) higher than that of its random equivalent $G^{R}$, then we can state that (part of) the decisions made by the entities that compose the network graph $G$ are non-random.

Real-world DCWN datasets: Our evaluations are performed on three real-world datasets (also referred to as traces in the following) that describe movements of entities in campus and city scenarios. The Dartmouth dataset [3] is a mobility trace of more than 1,000 individuals in the university campus, recorded over eight weeks using WiFi network access information. The USC dataset [2] is also a mobility trace in a campus scenario, comprising movement information of more than 4, 000 individuals over eight weeks, again collected through WiFi access. Finally, the San Francisco dataset [1] contains records of the mobility of 551 taxis in San Francisco, CA, 
USA, over one month, gathered through GPS logging at each cab in the urban area. For both the Dartmouth and USC traces, two individuals are assumed to generate a contact if they are using the same $\mathrm{WiFi}$ access point to connect to the wireless network on campus. In the San Francisco trace, two taxis are in contact if their distance is lower than 250 meters. Extensive experimental analysis in [17] shows that a distance of $250 \mathrm{~m}$ grants a $50 \%$ packet delivery ratio in urban environments, under common power levels (15-20 dBm) and with robust modulations (3-Mbps BPSK and 6-Mbps QPSK). In all cases, the contact events between two individuals are traced using start date of contact and its duration. Table 1 summarizes the features of the different datasets.

\section{MODELING}

This section introduces the basic operations performed on the dataset before RECAST is executed. Section 4.1 shows how we model the dynamic DCWN as a temporal aggregation graph. Section 4.2 details the algorithm we employ to obtain the equivalent random graph of a given temporal aggregation graph, and discusses how these two graphs compare in terms of clustering coefficient.

\subsection{Temporal Aggregation Graph}

As previously discussed, the datasets we employ list contacts among individuals or vehicles, and associate to each encounter a start time and a duration. In order to generate the temporal graph, we discretize time into steps of duration $\delta{ }^{1}$, and represent all the encounters occurring at time step $k$ as a graph $\mathcal{G}_{k}\left(\mathcal{V}_{k}, \mathcal{E}_{k}\right)$. The set of vertices $\mathcal{V}_{k}$ is composed of all network nodes (i.e., individuals or vehicles) involved in a contact during the $k$-th time step, while the edges in the set $\mathcal{E}_{k}$ represent the pairwise contacts during the same time step. Therefore, an edge between two nodes $i$ and $j$, with $i, j \in \mathcal{V}_{k}$, exists in $\mathcal{E}_{k}$ if $i$ and $j$ have met during time step $k$.

We can then define a time varying representation of the DCWN using a temporal accumulation graph $G_{t}=\left(V_{t}, E_{t}\right)$. Formally, $G_{t}=\left\{\mathcal{G}_{1} \cup \mathcal{G}_{2} \cup \ldots \cup \mathcal{G}_{t}\right\}$. As such, $V_{t}$ (respectively $E_{t}$ ) is the set of all vertices (edges) that appeared in the dataset between time 0 and time step $t$ included. Note that $G_{t}$ evolves over time and aggregates all the contacts in the dataset, thus comprising both social encounters and random encounters between network entities.

\subsection{Comparison with Random Graphs}

The first step to analyze the mobility patterns of the temporal accumulation graph $G_{t}$ is to build its random version $G_{t}^{R}$. The latter must feature similar topological characteristics as the original $G_{t}$ graph, i.e., the same number of nodes, edges, and empirical degree distribution. That way, the only difference between $G_{t}$ and $G_{t}^{R}$ lies in the way nodes are connected to each other. While in $G_{t}$ the nodes connect in a "semi-rational" way, in $G_{t}^{R}$ the connections happen in a purely random fashion. As we will show later, this difference can be leveraged to accurately determine the extent of randomness in the mobility of individuals in DCWNs.

We use two algorithms to to generate $G_{t}^{R}$ from $G_{t}$. The first algorithm, which we will call RND, is well known in the network science community [18]. The algorithm $G^{R}=\operatorname{RND}(G)$ receives a graph $G(V, E)$ as a parameter and returns a random graph $G^{R}\left(V, E^{R}\right)$ with the same topological characteristics as $G$, i.e., the same number of nodes, number of edges and degree distribution. In this way, we guarantee that the only difference between the real graph $G$ and $G^{R}$ is to whom each node connects, that is the focus of our analysis. Thus, given the degree distribution $D=$

\footnotetext{
${ }^{1}$ In our study, we considered a duration of $\delta=1$ day, since the data sets originate from human activities that feature daily routines.
}

$\left(d_{1}, d_{2}, \ldots, d_{n}\right)$ of $G$ with $n$ nodes, this algorithm assigns an edge between nodes $i$ and $j$ with probability $p_{i j}=\left(d_{i} \times d_{j}\right) / \sum_{k=1}^{|V|} d_{k}$.

The second algorithm, which we call T-RND, is an extension of RND and is able to generate random graphs from a temporal network $G_{t}$. As mentioned in Section 4.1, the temporal aggregation graph $G_{t}=\left\{\mathcal{G}_{1} \cup \mathcal{G}_{2} \cup \ldots \cup \mathcal{G}_{t}\right\}$ is the union of event graphs $\mathcal{G}_{t}$. Thus, the algorithm $G_{t}^{R}=\mathrm{T}-\operatorname{RND}\left(\mathcal{G}_{1}, \mathcal{G}_{2}, \ldots, \mathcal{G}_{t}\right)$ receives as parameters a set of consecutive event graphs $\mathcal{G}_{t}$ and returns a random temporal graph $G_{t}^{R}$. It constructs $G_{t}^{R}$ by executing RND in each event graph $\mathcal{G}_{t}$ and then aggregating it in a way that $G_{t}^{R}=\left\{\operatorname{RND}\left(\mathcal{G}_{1}\right) \cup \operatorname{RND}\left(\mathcal{G}_{2}\right) \cup \ldots \cup \operatorname{RND}\left(\mathcal{G}_{t}\right)\right\}$. In summary, both RND and T-RND randomly replicate the total number of contacts with distinct persons each individual had in a given time snapshot.

We first demonstrate the analytical power of random comparison in Figure 1, where we show the behavior of the clustering coefficient for graphs $G_{t}$ and $G_{t}^{R}$ over time for the three analyzed networks. As we have previously mentioned, the clustering coefficient is a good metric to differentiate social networks from random networks. As we observe in Figure 1-a, for the Dartmouth dataset, the clustering coefficient of $G_{t}$ and $G_{t}^{R}$ are different in orders of magnitude over the first days. However, as time goes by, their values get closer, as random encounters grow in number and tend to veil the social network structure. On the other hand, as we see in Figure 1-b, the clustering coefficients of $G_{t}$ and $G_{t}^{R}$ for the USC dataset are almost constant over time. However, the difference between them is not exceedingly high, since they have the same order of magnitude. We discuss these differences in detail later on.

Finally, as we observe in Figure 1-c, the clustering coefficients of the San Francisco networks are practically the same, being close to 1 . In fact, after a few hours, the network becomes similar to a clique, indicating a global high mobility, allowing each individual taxi encountering most of the other taxis at some point of the day. Formally, this indicates that $G_{t}$ and $G_{t}^{R}$ are very similar for the San Francisco dataset, i.e., the probability of random encounters is much higher than the probability of social contacts, what makes the San Francisco network similar to a random mobile network. This makes sense since taxis' decisions depend on occasional customers' requests rather than on routine mobility patterns of the driver.

\section{CLASSIFIER}

In this section, we describe the Random rElationship ClASsifier sTrategy (RECAST) we propose to differentiate relationships among individuals in a social network. More precisely, the purpose of RECAST is to tell apart random interactions from social-driven ones. To that end, Section 5.1 presents the DCWN features used by RECAST. Then, Section 5.2 introduces the RECAST algorithm and, finally, Section 5.3 discusses the results obtained by applying RECAST to the previously introduced datasets.

\subsection{Social Networks Features}

In order to identify social relationships, we must point out which features distinguish a social relationship from a random one. Indeed, two characteristics are always present in social relationships [19]:

1. Regularity. It is well known that social relationships are regular, in that they repeat over time. If two individuals are, for example, friends, co-workers, or daily commuters, they see each other regularly.

2. Similarity. It is expected that two individuals who share a social relationship have common acquaintances between 
Table 1: Datasets used in the presented investigations.

\begin{tabular}{|c|c|c|c|c|c|}
\hline Dataset & Local & Number of entities & Duration & Entities type & Avg. \# encounters/node/day \\
\hline Dartmouth [3] & University campus & 1156 & 2 months & Individuals & \\
\hline USC [2] & University campus & 4558 & 2 months & Individuals & 145.6 \\
\hline San Francisco [1] & City & 551 & 1 month & Cabs & 23.8 \\
\hline
\end{tabular}

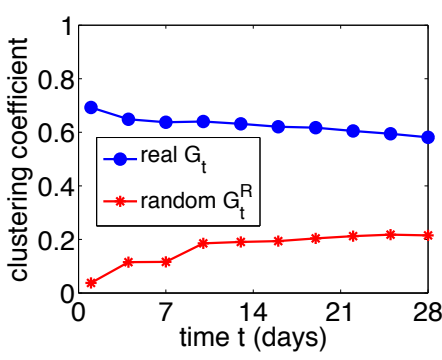

(a) Dartmouth

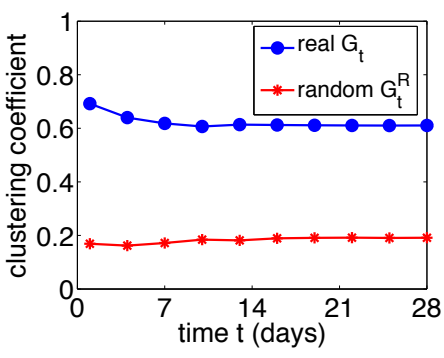

(b) USC

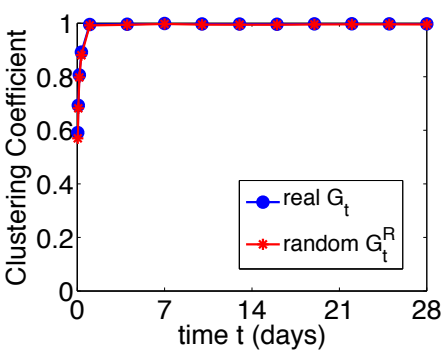

(c) San Francisco

Figure 1: Evolution of the clustering coefficient in the $G_{t}$ of the three datasets, and in their random equivalents $G_{t}^{R}$.

them. As an example, two individuals who share a large number of friends will most probably know each other as well.

Regularity and Similarity can be mapped into DCWN features that, in turn, can be computed from a contact dataset so as to identify what kind of relationship two individuals share. In the following, we discuss such features.

\subsubsection{Edge Persistence}

A complex network metric mapping of the Regularity of a relationship is the edge persistence. Basically, considering the set of event graphs $\left\{\mathcal{G}_{1}, \ldots, \mathcal{G}_{t}\right\}^{2}$, the edge persistence $\operatorname{per}_{t}(i, j)$ measures the percentage of times the edge $(i, j)$ occurred over the past discrete time steps $1,2, \ldots, t$. Formally, it is defined as $\operatorname{per}_{t}(i, j)=\frac{1}{t} \sum_{k=1}^{t} \mathbb{1}_{\left[(i, j) \in \mathcal{E}_{k}\right]}$, where $\mathbb{1}_{\left[(i, j) \in \mathcal{E}_{k}\right]}$ is an indicator function that assumes value 1 if the edge $(i, j)$ exists in $\mathcal{E}_{k}$ at time $k$, and 0 otherwise.

For instance, assuming that each day of the week is a time step, if Smith and Johnson met each other twice in a week, their edge persistence is the number of times they encountered, i.e., 2, divided by the total number of time steps, i.e., 7, or $\operatorname{per}_{t=7}($ Smith, Johnson $)=$ $2 / 7$. The edge persistence allows spotting regular relationships between two entities.

We show in Figure 2 (first column) the edge persistence as measured in the three datasets. Considering the set of event graphs $\left\{\mathcal{G}_{1}, \ldots, \mathcal{G}_{t}\right\}$ of all three real networks and their RNDgenerated random counterparts ${ }^{3}\left\{\operatorname{RND}\left(\mathcal{G}_{1}\right), \ldots, \operatorname{RND}\left(\mathcal{G}_{t}\right)\right\}$, we portray the complementary cumulative distribution function (CCDF) $\bar{F}_{p e r(i, j)}(x)=P\left[\operatorname{per}_{t}(i, j)>x\right]$. There, the time step is one day and each curve is obtained by analyzing four weeks of contacts, since $t=28$ corresponds to the length of the shortest considered dataset, i.e., the San Francisco one. From Figures 2-a and 2-c, we observe that the Dartmouth and USC networks have edge persistence distributions that significantly differ from those computed in their random equivalents. More precisely, while the CCDFs of random networks show an exponential decay, the individuals in the

\footnotetext{
${ }^{2}$ Note that edge persistence is computed over the set of graphs $\mathcal{G}_{t}$ and not over the temporal accumulation graph $G_{t}$.

${ }^{3}$ We generated five instances for every random graph and the cumulative distribution considers all of them.
}

real network tend to see each other regularly, i.e., for reasons beyond pure randomness, leading to a heavy-tailed distribution. Conversely, as from Figure 2-e, the encounters in the San Francisco dataset show an edge persistence similar to that obtained in the random equivalent graphs.

\subsubsection{Topological Overlap}

The Similarity of contacts can be mapped to the topological overlap feature of a complex network. This metric is extracted from the aggregated temporal graph $G_{t}$. The topological overlap $t_{t}(i, j)$ of a pair of nodes $i$ and $j$ is defined as the ratio of neighbors shared by two nodes, or, formally,

$$
t o_{t}(i, j)=\frac{\left|\left\{k \mid(i, k) \in E_{t}\right\} \cap\left\{k \mid(j, k) \in E_{t}\right\}\right|}{\left|\left\{k \mid(i, k) \in E_{t}\right\} \cup\left\{k \mid(j, k) \in E_{t}\right\}\right|} .
$$

In Figure 2 (second column), we show the $\operatorname{CCDF} \bar{F}_{t o(i, j)}(x)=$ $P\left[t_{t}(i, j)>x\right]$ of the topological overlap of the edges of the real networks $G_{t}$ and their respective random networks $G_{t}^{R}$, generated by the T-RND mechanism ${ }^{4}$. Again, we pick one day as the time step and consider four weeks of contacts (i.e., $t=28$ days). Similar to what occurred to the edge persistence, we note that the Dartmouth and USC network CCDFs significantly differ from their random counterparts, in Figures 2-b and 2-d. Indeed, pairs of individuals in these datasets share common neighbors in a way that could not happen randomly. Conversely, in Figure 2-f, the San Francisco network again behaves like a random contact network. Since all results indicate that the San Francisco network is random by nature, in the remainder of this work we will focus on the Dartmouth and the USC datasets.

\subsection{The RECAST algorithm}

We have seen that both the edge persistence and the topological overlap behave differently in contact graphs generated from realworld social networks and in their random equivalent graphs. We exploit such a diversity to identify which edges are consequences of random or social events. In particular, we propose a classification of relationships among network entities into four categories, depending on whether the edge corresponding to the relationship features random-like persistence and topological overlap. The four

\footnotetext{
${ }^{4}$ Again, we generated five instances for every random graph and the cumulative distribution considers all of them.
} 


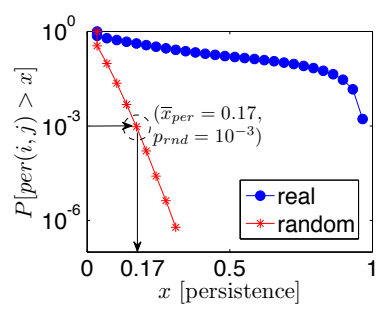

(a) Dartmouth

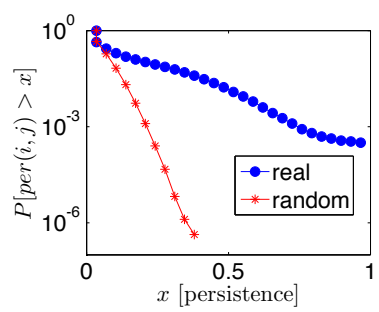

(c) USC

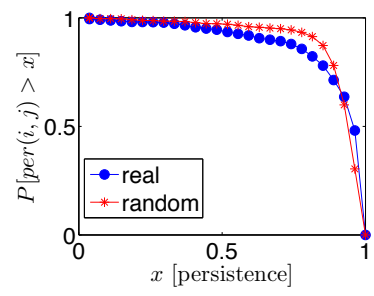

(e) San Francisco

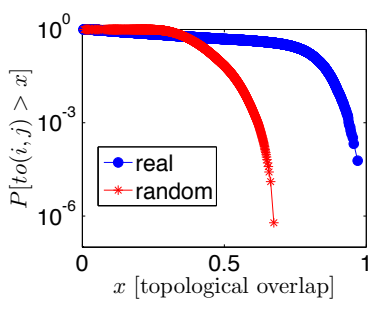

(b) Dartmouth

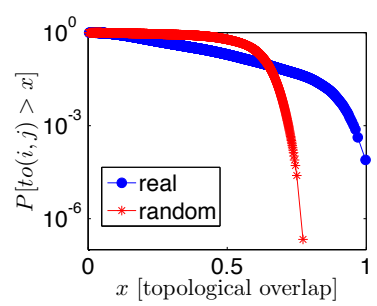

(d) USC

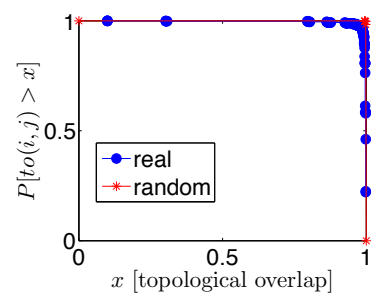

(f) San Francisco
Figure 2: The complementary cumulative distribution function of the edge persistence (a)(c)(e) and topological overlap (b)(d)(f) for the $G_{t}$ of the three datasets and for their random correspondents $G_{t}^{R}$ after four weeks.

classes of relationships are described in Table 2. A feature value is called "social" if there is an almost zero probability of this value being generated randomly. On the other hand, a feature value is called "random" if there is a significant probability of this value be generated randomly. In fact, as we explain in the following paragraphs, the unique parameter $p_{r n d}$ of RECAST defines if a given feature value is social or random.

Table 2: RECAST relationships classes.

\begin{tabular}{|c|c|c|}
\hline Class & Edge persistence & Topological overlap \\
\hline Friends & social & social \\
\hline Acquaintance & random & social \\
\hline Bridges & social & random \\
\hline Random & random & random \\
\hline
\end{tabular}

Relationships classified as Friends characterize pairs of individuals whose connection shows social edge persistence and topological overlap, i.e., who meet each other regularly and also tend to know the same people ${ }^{5}$. The Acquaintance class includes relationships among individuals sharing many common encounters, but not meeting often. As an example, friends of friends who see each

\footnotetext{
${ }^{5}$ It is worth mentioning that, although the friend terminology implies attachment among two individuals by affection or personal regard, we use it here to describe strong social ties in terms of regularity and similarity.
}

other once in a while, in occasions such as birthday parties, graduation ceremonies or weddings, would be classified as Acquaintance. The last social class is that of Bridges, characterizing pairs of individuals who see each other regularly, but do not share a large number of common acquaintances. E.g., the so-called familiar strangers, people who meet every day but do not really know each other (e.g., because they just commute between common home and work areas) are very likely to be classified as Bridges. Finally, when an edge is neither persistent nor characterized by topological overlap, it is considered the result of a random contact, and we classify it as Random.

In order to distinguish "social" from "random" values of the DCWN's features, we resort to the distributions we previously discussed. More precisely, we define a value $p_{r n d}$, the only parameter in RECAST, and we identify the feature value $\bar{x}$ for which $\bar{F}(\bar{x})=p_{\text {rnd }}$ for the random network $G_{t}^{R}$. The value $\bar{x}$ represents then a threshold, such that feature values higher than $\bar{x}$ occur with a probability lower than $p_{r n d}$ in a random network. If we set $p_{r n d}$ to some small value, we can finally state that feature values higher than $\bar{x}$ are very unlikely to occur in a random network, i.e., they are most probably due to actual social relationships. The parameter $p_{r n d}$ can also be seen as the expected classification error percentage. For instance, in Figure 2-a, consider $p_{r n d}=10^{-3}$. This gives a threshold $\bar{x}=0.17$, then all values higher than $\bar{x}=0.17$ will be classified as social. However, there is a $p_{r n d}=10^{-3}$ probability of randomly generating values that RECAST classified as social edges (i.e., false positives). In other words, we expect that $10^{-3}=0.1 \%$ of the edges classified as social to be, in fact, random. The full RECAST mechanism is described in Algorithm 1, where the criteria used in each classification are detailed. In this algorithm, index $t$ is omitted for per and to metrics for clarity purposes.

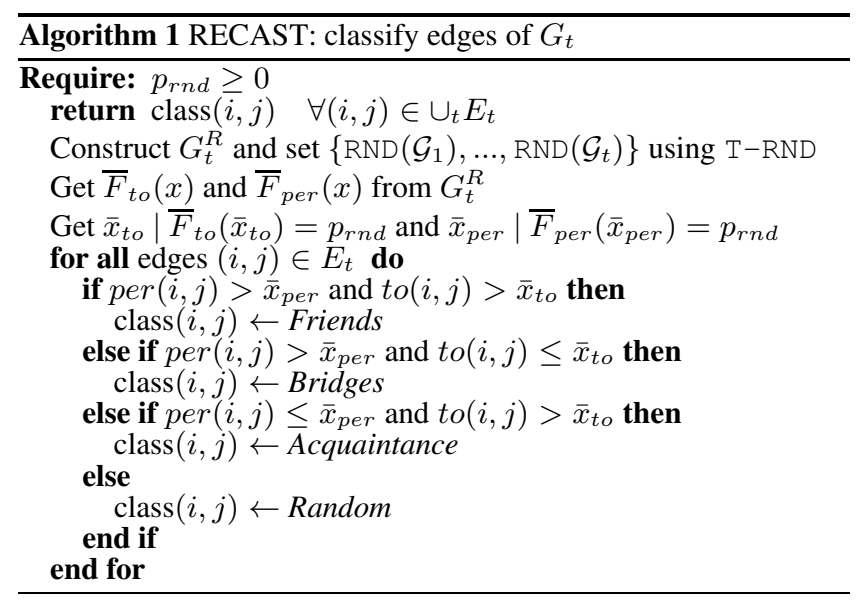

The complexity of RECAST is upper bounded by the construction of $G_{t}^{R}$ using T-RND, which is $O\left(t \times\left(\left|\mathcal{V}_{t}\right|+\left|\mathcal{E}_{t}^{R}\right|\right)\right)$, i.e., the minimum complexity for the generation of a degree sequencebased random graph available to date [18]. After the construction of $G_{t}^{R}$, the complexity of the classification mechanism is $O\left(\left|E_{t}^{R}\right| \times\left|V_{t}\right|\right)$, where $O\left(\left|V_{t}\right|\right)$ is the cost of computing the topological overlap of an edge.

\subsection{Classification results}

We apply RECAST to the Dartmouth and the USC networks. We are omitting the results for the San Francisco dataset, since, as previously stated, the random-like nature of taxi routes makes the analysis uninteresting, with all edges classified as Random. In Figure 3, we show the number of edges per class as a function of 


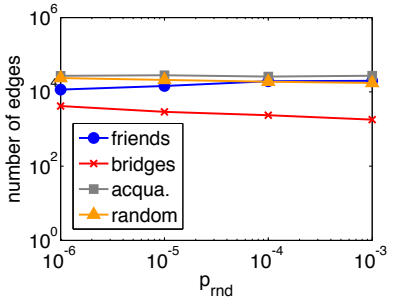

(a) Dartmouth

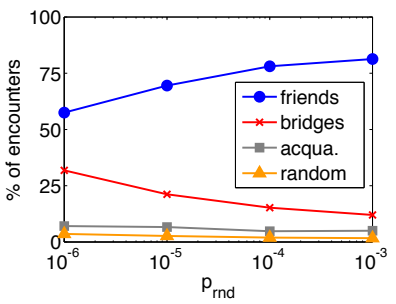

(c) Dartmouth

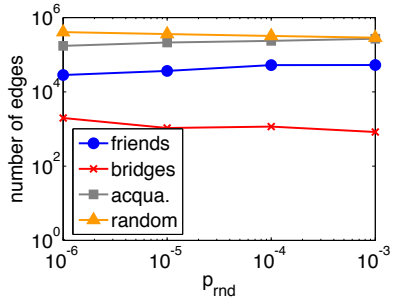

(b) USC

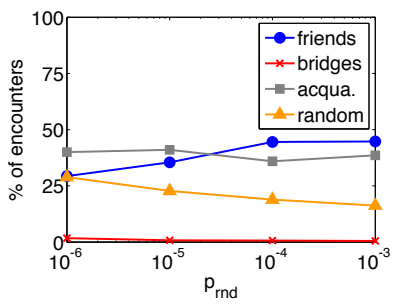

(d) USC
Figure 3: The number of edges (a)(b) and percentage of encounters $(c)(d)$ of a given class that appears in the first four weeks of data versus $p_{r n d}$.

the $p_{r n d}$ value. An initial and quite surprising observation is that, by varying $p_{r n d}$ through four orders of magnitude, the number of edges per class stays in the same magnitude. This shows that RECAST is robust with respect to $p_{r n d}$, i.e., it does not need a fine calibration of the parameter to return a consistent edge classification.

Secondly, in both datasets, the number of Bridges is orders of magnitude lower than the other classes, a clear indication that in the analyzed social networks regular connections among different communities are rare. Also the number of Friends edges is similar in the two networks, implying similar dynamics in tight relationships among individuals in the two campuses. This also agrees with the biological constraint on a social interaction that limits human social networks' size, i.e., the number of Friends relationships [20]. However, the two datasets differ when looking at the number of edges classified as Acquaintances and Random, that are one order of magnitude larger in USC than in Dartmouth. This is the result of the actual size of the two campuses, USC accounting for a population around ten times larger than that of Dartmouth. This aspect is also reflected by the size of the traces in Table 1 that clearly leads, in the USC network, to (i) many more Random contacts among individuals who do not actually know each other, but just happen to cross each other while strolling on campus, and (ii) an increased presence of strangers who happen to know the same people, leading to more Acquaintances edges.

The observations above are even more evident when observing the percentage of individual encounters of each type in the Dartmouth and USC networks. In Figure 3, we show the percentage of encounters of a given class that appear in the first four weeks of data for a given value of $p_{r n d}$. The percentage of Random encounters in the Dartmouth network is close to zero, varying from $1.7 \%$ to $3.6 \%$ as $p_{\text {rnd }}$ decreases. On the other hand, in the USC network, this percentage varies from $16 \%$ to $29 \%$. In fact, the proportion of Random encounters provides a good estimate of the probability of random decisions mentioned in Section 4. Thus, the USC network has a significantly higher tendency to evolve to a random topology than the Dartmouth network.

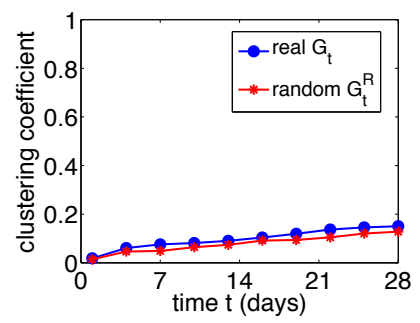

(a) Dartmouth

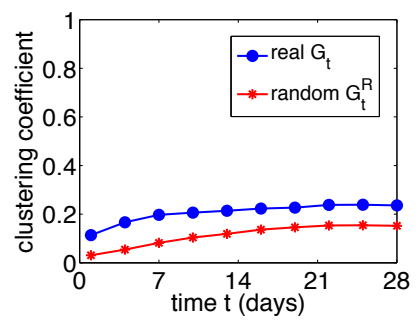

(b) USC
Figure 5: Evolution of the clustering coefficient of $G_{t}$ when only Random edges are present, compared to their random correspondents $G_{t}^{R}$.

The analysis is confirmed by Figure 4, portraying the snapshots of the Dartmouth and USC networks after two weeks of interactions, when considering only social edges (i.e., those classified as Friends, Acquaintances and Bridges) or only edges tagged as Random. Edges of the former networks in Figures 4-a and 4-c are distinguished by colors, according to the same code used in Figure 3 (Friends edges are in blue, Bridges in red, Acquaintances in gray, and Random in orange). The difference between the social-only networks and the random-only ones is striking. Social networks are characterized by a complex structure of Friends communities, linked to each other by Bridges and Acquaintances. More precisely, when comparing the Dartmouth and USC social networks, the former appears to be dominated by Friends interactions, while the sheer number of Acquaintances in the latter drives its graph structure.

Conversely, networks containing only Random edges do not show any structure and look like random graphs. A rigorous way to verify the randomness of such networks, and thus validate the efficiency of the RECAST classification, is to perform a clustering coefficient analysis. Figure 5 compares the clustering coefficients of the Dartmouth and USC networks $G_{t}$ when only Random edges are present against the same metric computed in their random counterparts $G_{t}^{R}$. The clustering coefficient, commonly employed to determine the actual randomness of a network, has very similar evolutions in $G_{t}$ and $G_{t}^{R}$ : this proves that the network of contacts tagged as Random by RECAST is actually a random network. Therefore, RECAST is able to extract from a real-world contact dataset edges that correspond to random encounters.

\section{RECAST APPLICATION}

In this section, we use the Dartmouth and USC contact traces to simulate an epidemic dissemination. We consider that the users communicate with each other in an opportunistic fashion, i.e., without any infrastructure and exchanging messages only when they are within physical proximity. Thus, if user $i$ wants to send a message to user $j$, he (she) has to deliver it personally to $j$ or has to ask other users to relay it for him (her), through a multi-hop carry-andforward path. Also, we consider the transfer to be epidemic, i.e., in order to reach $j$, user $i$ sends a message to all other users he (she) is in contact with at a given time $t$. The latter forward it to all of the users they later meet and so on, until user $j$ is reached. Such an epidemic approach allows us characterizing the lower bound on the delay required by the opportunistic transfer.

For both Dartmouth and USC contact traces, we use RECAST to classify the relationships between users over one month of contact data, which we refer to as the classifying stage. Then, we simulate the opportunistic transfer scenario above during the two following 


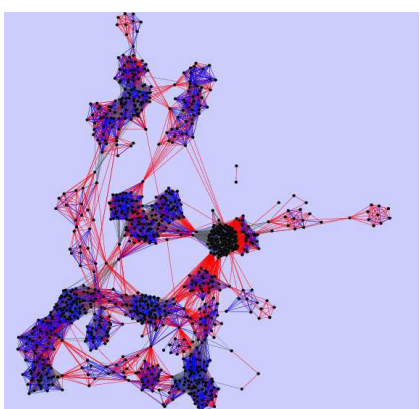

(a) Dartmouth, social edges

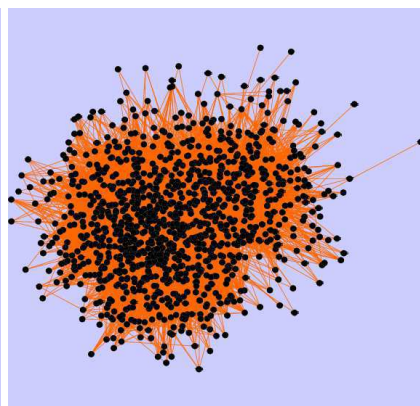

(b) Dartmouth, random edges

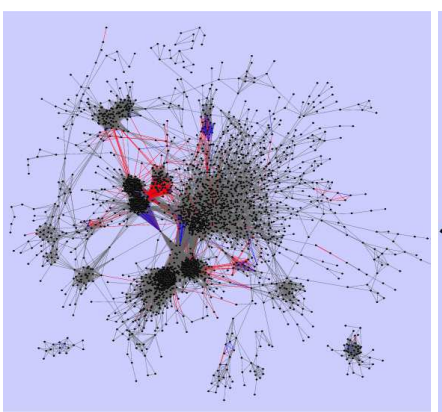

(c) USC, social edges

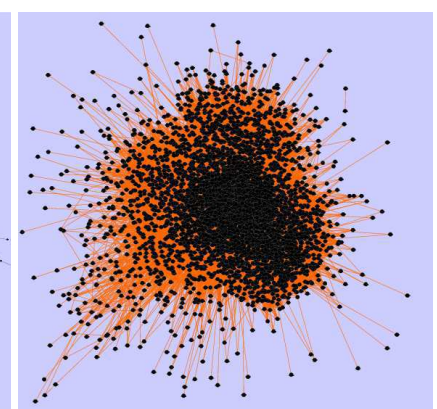

(d) USC, random edges

Figure 4: Snapshots of the Dartmouth and USC networks after two weeks of interactions, considering only the social edges and only the random edges. Friends edges are painted in blue, Bridges in red, Acquaintances in gray and Random in orange. This figure is best viewed in colors.

weeks, termed the routing stage, containing only future encounters, not known previously by RECAST. For each user $i$, we randomly pick a time $t_{0, i}$ within the first week of the routing stage for him/her to start the epidemic transfer process, and a destination user $j$. We leave one week for the message to reach its destination: if the message is not delivered by then, the transfer is deemed failed and the data lost.

In Figure 6, we show the overall forwarding efficiency. Figures 6-a and 6-b show the percentage of messages that were successfully delivered to their destinations in the Dartmouth and in the USC scenarios, respectively. Each bar represents one relationship shared by the message source and destination, and within each bar we depict the fraction of edges of a given class of relationship that was used to deliver the message. First, we observe that all the messages reached their destinations in the Dartmouth scenario, a consequence of the limited network size. Moreover, the edges classified as Friends were the most used to deliver the messages and the Random ones the least used. In fact, considering all the paths directed to Friends, less than $2 \%$ of the hops in these paths were given by users who share a Random relationship.

Different from the Dartmouth scenario, not all messages are delivered in the USC scenario. The lower delivery ratio is partially due to the larger network that is harder to navigate. However, that is not the only reason, and the social relationship between the source and destination significantly affects the probability of success. Socially connected pairs (tagged as Friends, Bridges and Acquaintances) can actually exchange data: $90 \%$ of the messages were successfully delivered to Friends, $92 \%$ to Bridges and $77 \%$ Acquaintances. If one wants to send a message to a Random contact in the USC scenario, there is only $44 \%$ of chances that this message will arrive successfully. Moreover, although the majority of the classified edges in the USC scenario are Random (see Figure 3), the majority of the hops in the paths are between users who share a social relationship. Considering all the paths directed to Friends, less than $13 \%$ of the hops in these paths were given by users who share a Random relationship.

In Figure 7, we show how much time it was necessary for the messages to reach their destinations. We grouped together all the routings from source user $i$ to destination user $j$ by the class of relationship $c$ that $i$ and $j$ share. Then, we cumulatively count how many destinations of the class $c$ are reached per each hour, considering the total number of routings that were performed between sources and destinations of class $c$. Observe that the expected time to reach a Random contact is significantly higher than the time needed to reach a social contact. Moreover, observe that the ma-

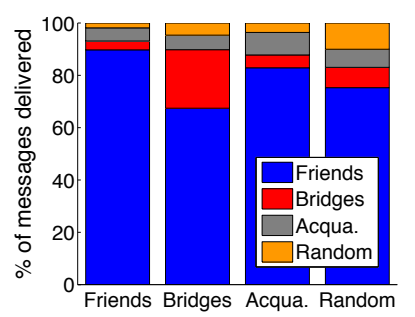

(a) Dartmouth

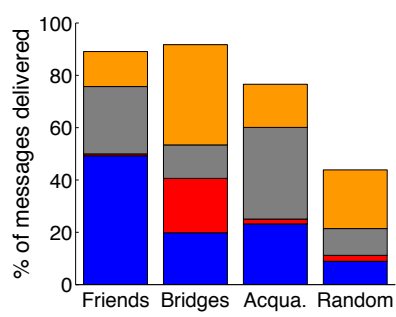

(b) USC
Figure 6: The forwarding efficiency when user $i$ sends a message to user $j$ in the opportunistic network, and $i$ and $j$ share a specific RECAST relationship. Within each bar we also show the fraction of edges of a given class of relationship that was used to deliver the message.

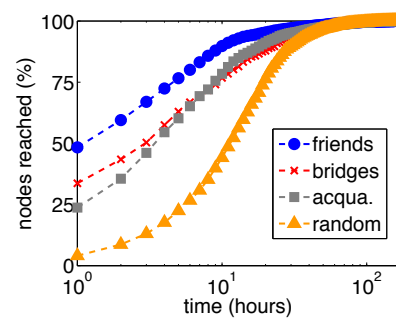

(a) Dartmouth

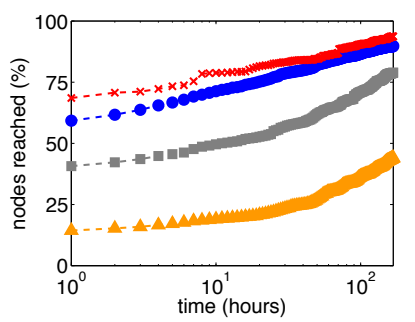

(b) USC
Figure 7: The $\%$ of users who were reached over the time.

jority of the messages sent to Friends arrive in the first hours for both scenarios.

It is not only the time it takes for a message to arrive at its destination that is relevant to the design of forwarding solutions for opportunistic networks. Another fundamental aspect is the number of hops required to reach the destination. In Figure 7, we show the cumulative distribution function (CDF) of the path lengths of messages between users $i$ and $j$ who share a determined class of relationship. Observe that the expected number of hops for a message to arrive at a Random contact is significantly higher than to arrive at a social contact. For the USC scenario, $89 \%, 92 \%$ and $81 \%$ of the routes to Friend, Bridge and Acquaintance destinations, respectively, have path lengths lower or equal to 3 . In the meanwhile, only $65 \%$ of the routes to Random destinations have path lengths 


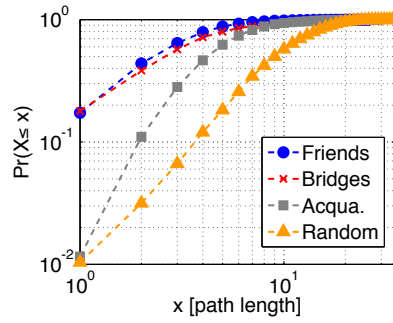

(a) Dartmouth

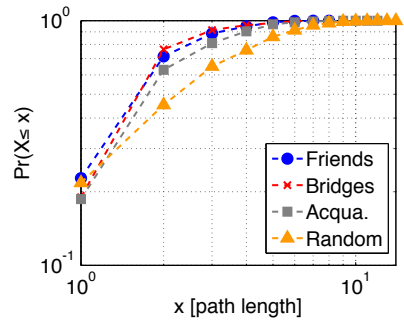

(b) USC
Figure 8: The histogram of the path lengths of messages between users $i$ and $j$ who share a determined class of relationship.

lower or equal to 3 . This difference is even more striking for the Dartmouth scenario, where $65 \%, 57 \%$ and $28 \%$ of the routes to Friend, Bridge and Acquaintance destinations, respectively, have path lengths lower or equal to 3 , and only $6 \%$ to Random destinations have path lengths lower o equal to 3 .

Overall, our results show how the RECAST classification allows identifying those who share social relationships with the sender, whose opportunistic paths are usually short and reliable. In fact, such paths usually pass through a few number of hops, mostly using social ties among users, and rarely leverage random encounters. As an intuitive corollary, reaching users that share some social relationship is significantly easier than attaining users one does not know, especially in large systems. These results may serve to leverage the performance of various routing solutions for opportunistic networks. If we previously know the class of relationship the destination share with the source, we also know the chances and the probable time the message will take to arrive.

\section{CONCLUSIONS}

The contribution of this paper is threefold. First, we modeled three real-world mobile user encounter datasets as temporal contact graphs and we proposed the use of random equivalent graphs to outline their hidden social structure. Our original approach shows that different mobility traces can yield completely different social structures, determined by diverse behaviors of the entities participating in the system. These results let us speculate that researchers should not generalize their results based on the analysis of a single dataset. Second, we proposed the RECAST strategy to lazily classify random and social relationships in temporal social networks, and demonstrate its simplicity and effectiveness. Third, we employed the RECAST classification to the case of epidemic opportunistic transfers, and showed its relevance towards the identification of faster, reliable paths leveraging social ties among users.

As future work, we plan to analyze other mobility scenarios, both real and synthetic. Moreover, it would be interesting to apply the RECAST to other social networks, such as communication networks from phone calls or SMSs.

\section{REFERENCES}

[1] A. Rojas, P. Branch, and G. Armitage, "Experimental validation of the random waypoint mobility model through a real world mobility trace for large geographical areas," in Proceedings of the 8th ACM MSWiM, MSWiM '05, (New York, NY, USA), pp. 174-177, ACM, 2005.

[2] W. jen Hsu and A. Helmy, "Impact: Investigation of mobile-user patterns across university campuses using wlan trace analysis," CoRR, vol. abs/cs/0508009, 2005.
[3] T. Henderson, D. Kotz, and I. Abyzov, "The changing usage of a mature campus-wide wireless network," in Proceedings of the 10th annual international conference on Mobile computing and networking, MobiCom '04, (New York, NY, USA), pp. 187-201, ACM, 2004.

[4] M. C. Gonzalez, C. A. Hidalgo, and A.-L. Barabasi, "Understanding individual human mobility patterns," Nature, vol. 453, pp. 779-782, 2008.

[5] J. Leskovec, L. Backstrom, R. Kumar, and A. Tomkins, "Microscopic evolution of social networks," in $K D D$ '08: Proceeding of the 14th ACM SIGKDD, (New York, NY, USA), pp. 462-470, ACM, 2008.

[6] E. Cho, S. A. Myers, and J. Leskovec, "Friendship and mobility: user movement in location-based social networks," in Proceedings of the 17th ACM SIGKDD, KDD '11, (New York, NY, USA), pp. 1082-1090, ACM, 2011.

[7] A. Barabasi and R. Albert, "Emergence of Scaling in Random Networks," Science, vol. 286, no. 5439, p. 509, 1999.

[8] R. Albert, H. Jeong, and A.-L. Barabási, "Diameter of the World Wide Web," Nature, vol. 401, pp. 130-131, September 1999.

[9] P. Erdös and A. Rényi, "On the evolution of random graphs," Publ. Math. Inst. Hung. Acad. Sci., vol. 7, p. 17, 1960.

[10] A. Mtibaa, M. May, C. Diot, and M. Ammar, "Peoplerank: Social opportunistic forwarding," in IEEE Infocom, 2010.

[11] W. Gao, Q. Li, B. Zhao, and G. Cao, "Multicasting in delay tolerant networks: a social network perspective," in ACM MobiHoc, 2009.

[12] M. Conti, R. Di Pietro, A. Gabrielli, L. V. Mancini, and A. Mei, "The Smallville Effect: Social Ties Make Mobile Networks More Secure Against the Node Capture Attack," in ACM MobiWac, 2010.

[13] A. G. Miklas, K. K. Gollu, K. K. W. Chan, S. Saroiu, K. P. Gummadi, and E. De Lara, "Exploiting social interactions in mobile systems," in Proceedings of the UbiComp '07, (Berlin, Heidelberg), pp. 409-428, Springer-Verlag, 2007.

[14] G. Zyba, G. M. Voelker, S. Ioannidis, and C. Diot, "Dissemination in opportunistic mobile ad-hoc networks: The power of the crowd," in Proceedings of IEEE INFOCOM 2011, pp. 1179-1187, IEEE, Apr. 2011.

[15] S. Milgram, The individual in a social world, ch. The Familiar Stranger: An Aspect of Urban Anonymity, pp. 51-53. Addison-Wesley, 1977.

[16] D. J. Watts and S. H. Strogatz, "Collective dynamics of "small-world" networks," Nature, vol. 393, pp. 440-442, 1998.

[17] F. Bai, D. Stancil, and H. Krishnan, "Toward understanding characteristics of dedicated short range communications (dsrc) from a perspective of vehicular network engineers.," in ACM MobiCom, 2010.

[18] F. Chung and L. Lu, "Connected Components in Random Graphs with Given Expected Degree Sequences," Annals of Combinatorics, vol. 6, pp. 125-145, Nov. 2002.

[19] C. A. Hidalgo and C. Rodriguez-Sickert, "The dynamics of a mobile phone network," Physica A: Statistical Mechanics and its Applications, vol. 387, no. 12, pp. 3017 - 3024, 2008.

[20] R. I. M. Dunbar, "The social brain hypothesis," Evol. Anthropol., vol. 6, no. 5, pp. 178-190, 1998. 